\title{
Reticulocyte Hemoglobin Content Helps Avoid Iron Overload in Hemodialysis Patients: A Retrospective Observational Study
}

\author{
DOMENICO CAPONE ${ }^{1}$, MAURO CATALDI ${ }^{1,2}$, MAURO VINCIGUERRA ${ }^{3}$, TERESA MOSCA ${ }^{1}$, \\ SALVATORE BARRETTA ${ }^{4}$, ANNALISA RAGOSTA ${ }^{4}$, ANIELLO SORRENTINO ${ }^{5}$, \\ ALESSANDRA VECCHIONE $^{5}$, LUCA BARRETTA ${ }^{5}$ and GIOVANNI TARANTINO ${ }^{6}$ \\ ${ }^{1}$ Integrated Care Department of Clinical Neurosciences, Anestesiology and Drug-Use, \\ Section of Clinical Pharmacology, "Federico II" University, Naples, Italy; \\ ${ }^{2}$ Division of Pharmacology, Department of Neuroscience, Reproductive and Odontostomatologic Sciences, \\ "Federico II" University of Naples, Naples, Italy; \\ ${ }^{3}$ Section of Nephrology, Santa Maria Delle Grazie Hospital, Pozzuoli, Italy; \\ ${ }^{4}$ Outpatients Clinic of Hemodialysis Dial Center s.r.l. Pomigliano D'Arco, Naples, Italy; \\ ${ }^{5}$ Diagnostic Center Kappa SRL Pomigliano D'Arco, Naples, Italy; \\ ${ }^{6}$ Department of Clinical Medicine and Surgery, "Federico II" University, Naples, Italy
}

\begin{abstract}
Background/Aim: Anemia in patients suffering from end-stage renal failure is currently treated with Erythropoiesis-Stimulating Agents (ESA). This treatment needs sufficient iron supplementation to avoid an inadequate dosage of ESA. Nowadays modern analytical instruments allow to accurately calculate the content of Hemoglobin $(\mathrm{Hb})$ in reticulocytes $(\mathrm{CHr})$, that can be used as a guide for prescribing patients with the appropriate amount of iron. Patients and Methods: Patients, undergoing hemodialysis, were retrospectively selected from the database and were divided in two groups: group A received intravenous (IV) iron and subcutaneously ESA, and their dosages were adjusted on the basis of the following parameters: $\mathrm{Hb}$, Mean corpuscular haemoglobin (MCH), $\mathrm{CHr}$ with consequent $\mathrm{MCH} / \mathrm{CHr}$ ratio and reticulocyte count determined by the ADVIA 120 Hematology System of Siemens; group B patients were administered IV iron and ESA monitoring iron storage, $\mathrm{Hb}$ and ferritin. The aforementioned parameters and the administered amount of iron and ESA were monitored at baseline, four and eight months from the begining of the study. Results: For ESA supplementation, no difference was
\end{abstract}

This article is freely accessible online.

Correspondence to: Dr. Giovanni Tarantino, Department of Clinical Medicine and Surgery, "Federico II" University, Via S. Pansini 5, 80131 Naples, Italy. Tel: +39 817462024, Fax: +39 817465462, email: tarantin@unina.it

Key Words: Reticulocyte hemoglobin content, anemia and iron supplementation, hemodialysis. observed between the groups at the various observed times. Despite similar Hb levels, the patients of group A needed significant lower doses of IV iron (-57.8\%) avoiding risks of organ toxicity and obtaining consequent cost saving of nearly $1 € /$ patient/month. Conclusion: The use of $\mathrm{CHr}$ and its related parameters allows the avoidance of overdosage of $I V$ iron, which can potentially damage organs, and the reduction of health care direct and indirect costs.

Patients suffering from End-Stage Renal Disease (ESRD) represent a major health care problem with a significant cost, should they undergo hemodialysis treatment. Patients lose up to $5-7 \mathrm{mg}$ of iron during each dialysis treatment and this is a primary cause of their iron-deficiency anemia $(1,2)$. There is also an increased need for iron supplementation to maintain $\mathrm{Hb}$ levels within the optimal range and maximize the response to ESA (3). As oral iron supplementation is often ineffective due to both patient non-compliance and gastrointestinal adverse effects, most dialysis patients receive IV iron to fill sufficient iron stores $(4,5)$. However, iron excess is stored in the liver causing organ toxicity and inflammation as well as an increased risk of infections $(6,7)$.

ESRD patients generally need to be given ESA and IV iron together to achieve the optimal $\mathrm{Hb}$ concentrations (4). The majority of patients on hemodialysis receive an IV/subcutaneous ESA dose during each dialysis section (8). Values of transferrin saturation $<20 \%$ and serum ferritin $<100 \mathrm{ng} / \mathrm{ml}$ require iron supplementation. (9). There are not specific protocols for IV iron supplementation. Previous clinical studies have suggested the administration of iron saccharate at a total dose of $1 \mathrm{~g} / 14$ days (two administrations of $500 \mathrm{mg}$ or five of $200 \mathrm{mg}$ ) (10) or iron gluconate at a total 
Table I. Characteristics of enrolled patients at baseline.

\begin{tabular}{lccc}
\hline & Group A & Group B & $p$-Value \\
\hline Number (M/F) & $15(8 / 7)$ & $17(8 / 9)$ & 0.803 \\
Age (years) & $70.0(62.0-73.7)$ & $73.0(64.2-77.7)$ & 0.199 \\
Duration of dialysis (years) & $4.0(3.0-6.5)$ & $3.0(2.0-6.0)$ & 0.392 \\
ESA dose (U/kg/patient/month) & $425.3(201.5-770.8)^{*}$ & $443.2(217.6-612.9)^{* *}$ & 0.682 \\
Hb (mg/dL) & $11.2 \pm 1.4$ & $11.6 \pm 1.0$ & 0.388 \\
Ferritin (ng/mL) & $286.0(168.5-337.2)$ & $230.0(159.1-395.8)$ & 0.737 \\
\hline
\end{tabular}

*Eight on epoetin zeta, 4 on darbepoetin alfa and 3 on methoxy polyethylene glycol-epoetin beta; **Five on epoetin zeta, 7 on darbepoetin alfa, 4 on methoxy polyethylene glycol-epoetin beta and 1 on epoetin beta.

dose of $1 \mathrm{~g} / \mathrm{month}$ (four administrations of $250 \mathrm{mg}$ ) (11). This approach leads to a suboptimal correction of anemia with consequent strong variations of $\mathrm{Hb}$ levels. These $\mathrm{Hb}$ variations are responsible for the continuous adjustments of iron and ESA dosages.

Reticulocyte count can represent a simple and cheap indirect way for the diagnosis of anemia. The count of erythrocytes by automatic methodologies is important and some issues of this procedure, such as the imprecision and inaccuracy due to manual count, have been eliminated. The quantitative measurement of fluorescence intensity rate represents a current parameter that completes the diagnostic value of reticulocyte count.

Thus, these modern analytical instruments allow to carefully calculate the $\mathrm{CHr}$ : $\mathrm{CHr}$ is related to $\mathrm{MCH}$ and the corresponding $\mathrm{MCH} / \mathrm{CHr}$ ratio is influenced both by the entity of erythropoiesis stimulation of the marrow and the iron availability for the $\mathrm{Hb}$ synthesis. In this study we have evaluated the usefulness of $\mathrm{CHr}$ (by using $\mathrm{MCH} / \mathrm{CHr}$ ratio) as a guide for the prescription of iron in order to reduce the fluctuations of $\mathrm{Hb}$ concentrations in patients undergoing hemodialysis. Testing ferritin levels is a routinely approach used to adjust iron supplementation in ESRD patients.

The aims of this work were: a) to compare the usefulness and appropriateness of $\mathrm{CHr}$, evaluated by $\mathrm{MCH} / \mathrm{CHr}$ ratio, versus ferritin in order to optimally set the iron supplementation in ESRD patients on dialysis; b) to consequently assess the cost saving analysis.

\section{Materials and Methods}

Population. Fifty Caucasian patients on stable hemodialysis treatment without high $\mathrm{C}$ reactive protein levels were retrospectively studied based on the data of their records. Eighteen patients were excluded not having filled the entire database, from which the laboratory parameters were drawn. Thirty two patients formed the final population. The selected patients were broken into two groups. The well matched characteristics of the population were reported in the Table I.
Table II. Amount of administered ESA (U/kg/patient/month) in both groups at observed times.

\begin{tabular}{cccc}
\hline & T0 & T4 & T8 \\
\hline Group A & $425.3(201.5-770.8)$ & $387.4(196.5-677.1)$ & $463.8(248.4-571.3)$ \\
Group B & $443.2(217.6-612.9)$ & $442.5(350.5-529.2)$ & $385.9(342.1-447.9)$ \\
\hline
\end{tabular}

ANOVA 2X2 Design: groups: $\mathrm{F}=0.99, p=0.323$; time: $\mathrm{F}=0.51, p=0.60$; groups*time: $\mathrm{F}=0.32, p=0.72$.

Laboratory parameters. Group A received iron and ESA, adjusting their dosages on the basis of the following parameters: $\mathrm{Hb}$, $\mathrm{MCH} / \mathrm{CHr}$ ratio, reticulocyte count (expressed as both absolute and relative values) by an automated hematology system count (ADVIA 120 Hematology System of Siemens Healthcare GmbH, Erlangen, Germany). It is a flow-cytometric analyser using lowangle and high-angle scatter for the determination of individual cell volume and $\mathrm{Hb}$ concentration (12). $\mathrm{CHr}$ is determined by measuring the volume and $\mathrm{Hb}$ concentration of each reticulocyte and it represents the mean value of $\mathrm{Hb}$ mass of each cell (13). This allows calculation of the following indexes: $\mathrm{MCH}, \mathrm{CHr}$ with consequent $\mathrm{MCH} / \mathrm{CHr}$ ratio and the reticulocyte production index (IPR). The $\mathrm{MCH} / \mathrm{CHr}$ ratio guides iron supplementation. A value $<1.0$ is indicative of physiologic availability of iron by the erythrone. A ratio $>1.0$ reveals iron deficiency and a consequent need for iron supplementation. Reticulocyte Production Index (RPI) addresses the administration of ESA; in particular RPI $<2.0$ indicates a reduced production of reticulocyte by the marrow with consequent need of ESA treatment; RPI $>2.0$ indicates a hyperplastic erythropoiesis and reduction until the withdrawal of ESA is performed. Vice versa, patients of group B were administered iron and ESA on the basis of the recommended guidelines for the treatment of anemia in chronic renal failure of the Italian Society of Nephrology (14) monitoring iron storage, $\mathrm{Hb}$ and ferritin. The afore mentioned parameters as well as the administered amount of iron and ESA were monitored at baseline, at four and eight months of the study.

Statistics. First of all, data of every studied variable were analyzed in order to assess the distribution by the Shapiro-Wilk (S-W) test. 
Table III. Hematologic parameters investigated in both groups at observed times.

\begin{tabular}{lccccccc}
\hline Group A & T0 & T4 & T8 & Group B & T0 & T4 & T8 \\
\hline $\mathrm{Hb}(\mathrm{mg} / \mathrm{dL})$ & $11.2 \pm 1.4$ & $11.1 \pm 1.4$ & $10.8 \pm 0.9$ & $\mathrm{Hb}(\mathrm{mg} / \mathrm{dL})$ & $11.6 \pm 1.0$ & $10.7 \pm 0.6$ & $11.6 \pm 0.6$ \\
$\mathrm{MCH} / \mathrm{CHr}$ & $0.95(0.94-0.96)$ & $0.91(0.88-0.93)$ & $0.88(0.87-0.92)$ & $\mathrm{MCH} / \mathrm{CHr}$ & $0.89(0.87-0.91)$ & $0.88(0.87-0.89)$ & $0.88(0.85-0.91)$ \\
$\mathrm{RPI}$ & $5.78(3.87-7.38)$ & $5.00(4.15-5.83)$ & $6.00(4.79-6.87)$ & RPI & $6.14(3.24-7.69)$ & $4.00(2.55-5.83)$ & $5.87(4.59-7.70)$ \\
Ferritin (ng/mL) & 286.0 & 116.0 & 153.0 & Ferritin & 230.0 & 213.0 & 285.5 \\
& $(168.5-337.2)$ & $(48.5-165.0)$ & $(48.5-198.0)$ & $(\mathrm{ng} / \mathrm{mL})$ & $(159.1-395.8)$ & $(171.7-345.3)$ & $(241.2-368.5)$ \\
\hline
\end{tabular}

Hb ANOVA 2X2 Design: groups: $\mathrm{F}=1.56, p=0.214$; time: $\mathrm{F}=2.17, p=0.120$; groups*time: $\mathrm{F}=2.93 ; p=0.06$; $\mathrm{MCH} / \mathrm{CHr}$ ANOVA $2 \mathrm{X} 2$ Design: groups: $\mathrm{F}=18.26, p \leq 0.001$; time: $\mathrm{F}=5.28, p=0.007$; groups*time: $\mathrm{F}=2.25, p=0.11$; RPI ANOVA $2 \mathrm{X} 2$ Design: groups: $\mathrm{F}=0.015, p=0.903$; time: $\mathrm{F}=2.19$, $p=0.118$; groups*time: $\mathrm{F}=0.132, p=0.876$; Ferritin ANOVA $2 \mathrm{X} 2$ Design: groups: $\mathrm{F}=9.66, p=0.0003$; time: $\mathrm{F}=3.739, p=0.028$; groups*time: $\mathrm{F}=2.563$, $p=0.083$

The normally distributed variables (S-W, $p>0.05)$ were expressed as mean $(\mathrm{M}) \pm$ standard deviation $(\mathrm{SD})$. Vice versa the abnormally distributed variables $(\mathrm{S}-\mathrm{W}, p<0.05)$ were expressed as median values (InterQuartile Range, IQR). To study frequencies, Chi squares were used. For the variable $\mathrm{Hb}$, which was normally distributed, the unpaired $t$-test was performed to assess the difference between two groups. When dealing with not normally distributed variables, the Mann-Whitney test was adopted in order to evaluate the difference between two groups.

An ANOVA $2 \times 2$ analysis was performed to evaluate differences between the two groups at the investigated times.

A $p$-value $<0.05$ was considered significant.

\section{Results}

In Table I the characteristics of the studied population at baseline were reported.

First of all, concerning the ESA supplementation, no difference was observed between groups and at the observed times (Table II). Stable values of $\mathrm{Hb}$ were present in both groups at the observed times and no significant difference was found between the groups A and B (Table III). Despite these similar $\mathrm{Hb}$ levels, the patients of group A needed significant lower doses of IV trivalent iron (-57.8\%). In fact, the total amount of administered iron in patients of groups $\mathrm{A}$ and B were 11,500 and 19,875 mg, respectively. For ferritin, although the basal values in both groups were similar, in the course of treatment its values tended to significantly decrease in the group A, confirming the minor need for iron supplementation. Accordingly, $\mathrm{MCH} / \mathrm{CHr}$ baseline values were superior to those of group B, with a trend to decrease during the follow up.

When analyzing the RPI values, no difference was observed between groups and regards to times, in agreement with the used doses of ESA.

Furthermore, a pharmacoeconomic evaluation showed a reduction in the cost of iron therapy of nearly $1 € /$ patient/ month.

\section{Discussion}

The loss of blood due to hemodialysis procedures but also laboratory testing is responsible for the anemia that usually is normocytic, normochromic and slightly regenerative. This condition needs ESA therapy, which should be associated with the administration of iron, as its efficacy is strongly linked to the adequate supplementation of this important trace metal. Optimal iron dosage is mandatory in order to avoid inappropriate ectopic storage (particularly liver, spleen, hearth, endocrine system) with related specific organ injury (15). In fact, overdose leads to an epidemic overload of iron in the ESRD population; IV iron bypasses the biological safeguards for the transport and handling of iron and helps to intensify chronic kidney disease-associated oxidative stress and inflammation. As a consequence, indiscriminate use of IV iron can accelerate cardiovascular disease, promote microbial infections, aggravate eventual viral hepatitis, and worsen diabetes and diabetic complications in such patients. For these reasons IV iron should be judiciously used in this vulnerable population (6).

Thus, adequate supplementation of both ESA and iron is central to reaching the $\mathrm{Hb}$ target concentrations. With regard to iron supplementation, IV iron is required as iron absorption is reduced along the gastro-intestinal tract due to elevation of hepcidin causing iron deficiency in ESRD patients. Even slight excess iron as a result of prolonged exposure can lead to toxicity. This approach could seem to be exaggerated, but it is mandatory to prevent long-term complications of iron overload and maintain serum iron and total body iron levels within a normal range. In this regards, the $\mathrm{CHr}$ could represent a valid diagnostic alternative although conflicting results exist in the literature. In fact, a previous study reported that the analysis of $\mathrm{CHr}$ is not superior to $\mathrm{MCH}$ when screening for iron deficiency in elderly anemic hospitalized patients (16). Conversely, other investigations carried out in animals and humans demonstrated an important predictive 
role of $\mathrm{CHr}$ in the diagnosis of iron deficiency (17). In our population, patients of group A required minor iron supplementation based on this recent analytical approach, and this finding is in agreement with the latter reported study.

Administering the ideal iron dosage avoids important fluctuations in ESA dosage, identifies no-responders and improves health care costs. The cost reduction, due to tailored iron therapy, could be considered an apparently inconsistent issue but, taking into account the large number of patients on haemodialysis in Italy and the prolonged mean time of treatment (various years), this saving is noteworthy. Direct medical costs (laboratory tests including also false positive or true positive results, instrumental measurements and hospital services) should be further added to the cost of drug prescriptions. Finally, indirect costs such as those of morbidity and mortality should be not overlooked (18).

Medical errors is the third leading cause of death in the United States, after heart diseases and cancer, according to recently published findings (19), burdened by skyrocketing health care costs. Medication errors can occur in deciding which medicine and dosage regimen to use. Errors in prescribing include ineffective under-prescribing and inappropriate over-prescribing (20).

A limitation of this study could be the relatively small sample size, although a prolonged time of observation should be considered a strength.

In conclusion, the use of erythrocyte indexes and their related parameters allows avoidance of over-dosage of IV iron, which can potentially damage organs, and reduction in health care direct and indirect costs.

\section{References}

1 Gupta A, Amin NB, Besarab A, Vogel SE, Divine GW, Yee J and Anandan JV: Dialysate iron therapy: infusion of soluble ferric pyrophosphate via the dialysate during the hemodialysis. Kidney Int 55: 1891-1898, 1999.

2 Gupta A and Crumbliss AL: Treatment of iron deficiency anemia: are monomeric iron compounds suitable for parenteral administration? J Lab Clin Med 136: 371-378, 2000.

3 Eschbach JW: State of the art Lecture. The future of r-HuEPO. Nephrol Dial Transplant 10(suppl 2): 96-109, 1995.

4 Fishbane S, Frel GL and Maesaka J: Reduction in human erythropoietin doses by the use of chronic intravenoue iron supplementation. Am J. Kidney Dis 26: 41-46, 1995.

5 Sunder-Plassmann G and Horl WH: Iron metabolism and iron substitution during erythropoietin therapy. Clin Investigation 72 : S11-S15, 1994.

6 Vaziri ND: Toxic effects of IV iron preparations in CKD patients. Nephrol News Issues 28: 4-5, 2014.

7 Fishbane S, Mathew A and Vaziri ND: Iron toxicy: relevance for dialysis patients. Nephrol Dial Transplant 29: 255-259, 2014.
8 Kaufman JS, Reda DJ, Fye CL, Goldfarb DS, Henderson WG, Kleinman JG and Vaamonde CA: Subcutaneous compared with intravenous epoetin in patients receiving hemodialysis. N Engl J Med 339: 578:583, 1998.

9 Locatelli F, Covic A, Eckardt K-U, Wiecek A and Vanholder R: Anemia management in patients with chronic kidney disease: a position statement by the Anemia Working Group of European Renal best Practice (ERBP). Nephrol Dial Transplant 24: 348354, 2009.

10 Van Wick DB, Roppolo M, Martinez CO, Mazey RM and McMurray S: A randomized, controlled trial comparing IV iron sucrose to oral iron in anemic patients with nondialysisdependent CKD. Kidney Int 68: 2846-2856, 2005.

11 Agarwal R, Rizkala AR, Bastani B, Kaskas MO, Leehey DJ and Besarab A: A randomized controlled trial of oral versus intravenous iron in chronic kidney disease. Am J Nephrol 26: 445-454, 2006.

12 Kotisaari S, Romppanem J, Penttila I and Punnonen K: The Advia 120 red blood cell and reticulocyte indices are useful in diagnosis of iron-deficiency anemia. Eur J Haematol 68: 150156, 2002.

13 Brugnara C, Hipp Mj, Irving PJ, Lathrop H, Lee PA, Minchello EM and Winkelman J: Automated reticulocyte counting and measurement of reticulocyte cellular indices. Evaluation of the Miles $\mathrm{H}^{*} 3$ blood analyzer. Am J Clin Pathol 102: 623-632, 1994.

14 Brancaccio D, Canavese C, Carozzi S, Cianciaruso B, Panzetta $\mathrm{G}$ and Piccoli A: Guidelines for the treatment of anemia in chronic renal failure. Giornale Italiano di Nefrologia 20(suppl 24): S61-S82, 2003.

15 Yamano N, Ikeda Y, Sakama M, Izawa-Ishizawa Y, Kihira Y, Ishizawa K, Miyamoto L, Tomita S, Tsuchiya $\mathrm{K}$ and Tamaki K: A long-term high-fat diet changes iron distribution in the body, increasing iron accumulation specifically in the mouse spleen. $\mathrm{J}$ Nutr Sci Vitaminol 61: 20-27, 2015.

16 Karlsson T: Comparative evaluation of the reticulocyte hemoglobin content assay when screening for iron deficiency in elderly anemic patients. Anemia 925907: 1-3, 2011.

17 Buttarello M, Pajola R, Novello E, Rebeschini M, Cantaro S, Oliosi F, Naso A and Plebani M: Diagnosis of iron deficiency in patients undergoing hemodialysis. Am J Clin Pathol 133: 949954, 2010.

18 Rice DP: Cost-of-illness studies: fact or fiction? Lancet 344: 1519-1520, 1994.

19 Makary MA and Daniel M: Medical error-the third leading cause of death in the US. BMJ 353: i2139, 2016.

20 Aronson JK: Medication errors: what they are, how they happen, and how to avoid them. QJM 102: 513-521, 2009. 